\title{
Human T Cell Lymphotropic Virus Type 1 Infection Among U.S. Thalassemia Patients
}

\author{
William M. Switzer, Anupama Shankar, ${ }^{1}$ Sean R. Trimble, ${ }^{2}$ Alexis A. Thompson, ${ }^{3}$ \\ Patricia J. Giardina, ${ }^{4}$ Alan R. Cohen, ${ }^{5}$ Thomas D. Coates, ${ }^{6}$ Elliott Vichinsky, \\ Ellis J. Neufeld, ${ }^{8}$ Jeanne M. Boudreaux, ${ }^{9}$ and Walid Heneine ${ }^{1}$
}

\begin{abstract}
Thalassemia is an inherited genetic disorder requiring multiple transfusions to treat anemia caused by low hemoglobin levels. Thus, thalassemia patients are at risk for infection with blood-borne pathogens, including human T cell lymphotropic viruses (HTLV) that are transmitted by transfusion of cellular blood products. Here, we examined the prevalence of HTLV among 234 U.S. thalassemia patients using sera collected in 2008. Sera were tested for antibodies to HTLV-1/2 using enzyme immunoassay (EIA) and a confirmatory western blot (WB) that differentiates between HTLV-1 and HTLV-2. Demographic information and clinical information were collected at study enrollment, including HIV and hepatitis C virus (HCV) status. Three patients (1.3\%) were WB positive; two were HTLV-1 and one could not be serotyped as HTLV-1/2. All three HTLV-positive persons were HIV-1 negative and one was HCV seropositive. The HTLV seroprevalence was higher than that of HIV-1 (0.85\%) and lower than HCV (18.8\%) in this population. All three patients (ages 26-46 years) were diagnosed with $\beta$-thalassemia shortly after birth and have since been receiving multiple transfusions annually. Two of the HTLV-positive patients confirmed receiving transfusions before HTLV blood screening was implemented in 1988. We identified a substantial HTLV-1 seroprevalence in U.S. thalassemia patients that is much greater than that seen in blood donors. Our findings highlight the importance of HTLV testing of patients with thalassemia and other diseases requiring multiple transfusions, especially in recipients of unscreened transfusions. In addition, appropriate counseling and follow-up of HTLV-infected patients are warranted.
\end{abstract}

$\mathbf{H}$ UMAN T-LYMPHOTROPIC VIRUS type 1 (HTLV-1) is the causative agent of adult $\mathrm{T}$ cell leukemia (ATL) and HTLV-1-associated myelopathy/tropical spastic paraperesis (HAM/TSP) and other inflammatory diseases in about $2-5 \%$ of those infected. ${ }^{1}$ HTLV-1 is endemic in the Caribbean, Japan, and parts of Africa and Brazil and is spread sexually, from mother to child, and by exposure to blood through transfusions and intravenous drug use (IDU). ${ }^{1}$ HTLV-2 is closely related to HTLV-1 and is endemic in IDUs and American Indian populations. HTLV-2 is transmitted in a manner similar to HTLV-1 but has been linked only to mild and infre- quent HAM/TSP and some inflammatory diseases. ${ }^{1}$ Transmission of HTLV-1 and HTLV-2 by blood transfusion occurs with transfusion of cellular blood products (whole blood, red blood cells, and platelets) but not with the plasma fraction or plasma derivatives from HTLV-1-infected blood. ${ }^{2}$ Transmission rates of $20-63 \%$ have been reported in recipients of HTLV-1-infected cellular components. ${ }^{2,3}$ The most recent prevalence of HTLV-1 among U.S. blood donations in 2009 was estimated to be about $3 \times 10^{-3} \cdot{ }^{4-6}$ Serological screening of U.S. blood donors was initiated in 1988 and has been effective in reducing transfusion transmission of HTLV-1 to $\sim 1$ per 4

\footnotetext{
${ }^{1}$ Laboratory Branch, Division of HIV/AIDS Prevention, National Center for HIV, Hepatitis, STD, and TB Prevention, Centers for Disease Control and Prevention, Atlanta, Georgia.

${ }^{2}$ Epidemiology and Surveillance Branch, Division of Blood Disorders, National Center on Birth Defects and Developmental Disabilities, Centers for Disease Control and Prevention, Atlanta, Georgia.

${ }^{3}$ Ann \& Robert H. Lurie Children's Hospital of Chicago, Chicago, Illinois.

${ }^{4}$ Weill Medical College of Cornell University, New York, New York.

${ }^{5}$ Children's Hospital of Philadelphia, Philadelphia, Pennsylvania.

${ }^{6}$ Children's Hospital of Los Angeles, Los Angeles, California.

${ }^{7}$ Children's Hospital of Oakland, Oakland, California.

${ }^{8}$ Children's Hospital Boston, Boston, Massachusetts.

${ }^{9}$ Children's Healthcare of Atlanta, Atlanta, Georgia.
} 
million donations. ${ }^{6}$ Frequent recipients of cellular blood products over long periods of time may be at increased risk of acquiring HTLV, especially if such transfusions occurred before the implementation of blood screening.

Thalassemias are a group of inherited blood disorders in persons mostly of Mediterranean and Asian descent with defective $\alpha$-globin or $\beta$-globin genes, resulting in impaired hemoglobin synthesis and anemia. Patients with thalassemia major require lifelong regular red blood cell transfusions, while patients with symptomatic thalassemia intermedia syndromes often require intermittent transfusion during their lives. Donor exposure over decades in thalassemia patients is as high, or higher, than that for other transfusion recipients. Here, we report HTLV infection among U.S. patients with thalassemia and discuss the implications of the findings.

In this study we tested de-identified sera collected during 2008 from 234 patients enrolled in a Centers for Disease Control (CDC)-sponsored thalassemia surveillance study at seven U.S. treatment centers (Table 1). Institutional Review Boards at CDC and each treatment center approved the study and future testing of archived specimens. A nonresearch determination was obtained at CDC to test anonymized, unlinked samples for HTLV. Demographic and clinical information was available from questionnaire data obtained at enrollment. Participants were enrolled into the study from 2004 to 2010 and were composed of almost equal numbers of males and females and persons of white and Asian descent (Table 1$)$. The majority of participants $(80 \%)$ were diagnosed

Table 1. Characteristics of Thalassemia Treatment Center Participants $(n=234), 2004-2010$

\begin{tabular}{|c|c|}
\hline & Number $(\%)$ \\
\hline \multicolumn{2}{|l|}{ Sex } \\
\hline Male & $111(47.4)$ \\
\hline Female & $123(52.6)$ \\
\hline \multicolumn{2}{|l|}{ Race } \\
\hline Black or African-American & $8(3.4)$ \\
\hline White & $97(41.5)$ \\
\hline Asian & $128(54.7)$ \\
\hline Other & $1(0.4)$ \\
\hline \multicolumn{2}{|l|}{ Diagnosis } \\
\hline $\begin{array}{l}\beta \text {-Thalassemia, transfused } \geq 8 \text { times } \\
\text { in previous } 12 \text { months }\end{array}$ & $178(76.1)$ \\
\hline $\begin{array}{l}\beta \text {-Thalassemia, with a history of } \\
\text { transfusion but }<8 \text { times in previous } \\
12 \text { months }\end{array}$ & $4(1.7)$ \\
\hline$\beta$-Thalassemia, never transfused & $11(4.7)$ \\
\hline$\alpha$-Thalassemia (hemoglobin $\mathrm{H}$ disease) & $7(2.9)$ \\
\hline$\alpha$-Thalassemia (hemoglobin H-Constant & $9(3.9)$ \\
\hline \multicolumn{2}{|l|}{ Spring disease) } \\
\hline $\begin{array}{l}\text { E- } \beta \text {-Thalassemia, transfused } \geq 8 \text { times in } \\
\text { previous } 12 \text { months }\end{array}$ & $10(4.2)$ \\
\hline $\begin{array}{l}\text { E- } \beta \text {-Thalassemia, with a history of } \\
\text { transfusion but }<8 \text { times in previous } \\
12 \text { months }\end{array}$ & $2(0.9)$ \\
\hline E- $\beta$-Thalassemia, never transfused & $6(2.6)$ \\
\hline Homozygous $\alpha$-thalassemia & $1(0.4)$ \\
\hline Other $^{\mathrm{a}}$ & $6(2.6)$ \\
\hline
\end{tabular}

${ }^{a}$ Hemoglobin H-Constant Spring with hemoglobin E trait, C-ßthalassemia, $\alpha$-thalassemia hemoglobin Dartmouth compound. with $\mathrm{B}$-thalassemia and reported chronic transfusion during the past year (Table 1).

Serologic testing for HTLV-1 and HTLV-2 was performed by using an HTLV-1/2 enzyme immunoassay (EIA) (HTLVI/II ELISA v4.0, MP Biomedicals, Solon, OH) followed by confirmation with an HTLV-1-based western blot (WB; HTLV Blot v2.4, MP Biomedicals, Singapore) assay that also includes type-specific antigens that differentiate between HTLV-1 and HTLV-2. Both the HTLV EIA and WB assays are used worldwide but are not currently licensed in the United States and were used for research purposes only. Information on HIV-1 and hepatitis $\mathrm{C}$ virus (HCV) infection was available from testing performed at enrollment during the study.

Five sera were HTLV EIA positive. Three were strongly EIA reactive (Table 2), and these were all found to be WB positive. Two of these three persons (Thal-18 and Thal-191) were HTLV-1 positive and one (Thal-237) showed no reactivity to either the HTLV-1 or HTLV-2 type specific recombinant proteins and was considered HTLV positive but untypeable (Fig. 1). Peripheral blood lymphocytes were not collected from this cohort to allow HTLV genotyping by polymerase chain reaction (PCR). Thus, the overall HTLV seroprevalence in this patient population was $1.3 \%(0.33-4.0$, 95\% CI).

All three WB-positive persons were born in the United States. The two HTLV-1-positive persons (Thal-18 and Thal191) were both females ages 48 and 26 years old, and received 17 and 15 blood transfusions during the past year, respectively (Table 2). Patient Thal-237 was a 40-year-old male who received 20 transfusions in the previous year. All reported receiving chronic blood product transfusion and all were diagnosed with $\beta$-thalassemia when less than 1 year old, and thus received transfusions before HTLV screening began in 1988. All three patients were HIV-1 negative while only patient Thal-191 was seropositive for HCV (Table 2). The overall cohort prevalence is $0.85 \%(0.14-3.37,95 \% \mathrm{CI})$ and $18.8 \%$ (14.1-24.5, 95\% CI) for HIV and HCV, respectively. Thus, the prevalence of HTLV in this cohort was 0.65 times higher than HIV-1 and 14 times less than HCV. All persons in the cohort with HCV and HIV-1 were positive at enrollment in 2004.

The $1.3 \%$ HTLV-1 prevalence we detected in the thalassemia patients is significantly higher than that seen in U.S. blood donors ( $p<0.0001$, Chi-square goodness of fit test). The relatively high HTLV prevalence cannot be explained by acquisition of HTLV from HTLV-screened transfusions, since the frequency of HTLV infection from such transfusions is exceedingly rare, about 1 in 2.3 million. ${ }^{6}$ Assuming each patient received 12 transfusions per year from different donors since the initiation of HTLV blood screening in 1988, the cumulative number of transfusions received in the 234 patients until 2008 would be around 56,160 transfusions, which is far too low to explain three HTLV infections. Therefore, other sources of infection may be inferred, the most likely being unscreened blood product transfusion before 1988. This is consistent with the reported chronic transfusions and age distribution in this cohort. Indeed, 136 persons (58.1\%) were born before 1989, of which the majority $(117 / 136 ; 86 \%)$ received transfusions prior to the implementation of routine HTLV screening of blood donors. The majority began receiving transfusions at thalassemia diagnosis less than a year old, including persons Thal18 and Thal-237. Thus, the HTLV prevalence in this subgroup of patients receiving transfusions before 1988 is $1.71 \%(2 / 117$; 
Table 2. Clinical and Demographic Information for Thalassemia Patients Seropositive to Human T-Lymphotropic Virus Type 1

\begin{tabular}{|c|c|c|c|c|c|c|c|c|c|}
\hline & $\begin{array}{c}\text { HTLV-1 } \\
\text { EIA S/CO }\end{array}$ & $\begin{array}{l}\text { HTLV WB } \\
\text { profile }\end{array}$ & $H I V$ & $\mathrm{HCV}$ & $\begin{array}{c}\text { Age } \\
\text { (years) }\end{array}$ & Gender & $\begin{array}{c}\text { No. } \\
\text { transfusions }{ }^{\mathrm{c}}\end{array}$ & $\begin{array}{c}\text { Age at diagnosis } \\
\text { (months) }\end{array}$ & $\begin{array}{l}\text { Marital } \\
\text { status }\end{array}$ \\
\hline Thal-191 & * & HTLV-1 & Neg & Pos & 26 & $\mathrm{~F}$ & 15 & 5 & Married \\
\hline Thal-18 & 7.77 & HTLV-1 & Neg & Neg & 48 & $\mathrm{~F}$ & 17 & 9 & Single \\
\hline Thal-237 & * & $\begin{array}{l}\text { HTLV-positive, } \\
\text { untypeable }\end{array}$ & Neg & Neg & 40 & M & 20 & 8 & Partnered \\
\hline
\end{tabular}

aS/CO, signal to cut-off ratio; values $\geq 1.0$ are considered reactive.

${ }^{\mathrm{b}}$ Age at enrollment.

${ }^{\mathrm{C}}$ Number of transfusions in past 12 months.

*Optical densities were above the dynamic range of the assay and S/CO could not be determined but also indicate strong seroreactivity.

HTLV-1, human T-lymphotropic virus type 1; EIA, enzyme immunoassay; WB, western blot; HCV, hepatitis C virus.

0.3-6.65\%, 95\% CI). Patient Thal-191 did not report the age of her first transfusion but most likely required transfusions at an early age, given a history of chronic transfusions. Similarly, the majority (75\%) of HCV infections occurred prior to 1992 and both HIV-1 infections occurred before 1985 when all blood donations were screened for HCV and HIV, respectively, further supporting a cohort effect of viral infection in older patients in our study population.

Alternative sources of infection, including sex with HTLVinfected partners, blood-borne transmission via IDU, and infection occurring outside the United States in countries where blood is not routinely screened for HTLV, cannot be fully excluded, although they do not readily explain the relatively high HTLV prevalence. Our results warrant further investigation in this patient population to better define the HTLV prevalence and viral types. These investigations can be aided by molecular testing to differentiate between HTLV-1 and HTLV-2, particularly when WB testing cannot type the infection, as was the case in one patient in our study. Unfortunately, the unlinked study design of our study did not allow us to obtain fresh blood specimens to investigate the evolutionary history of HTLV-1 in these three persons. Since HTLV-1 is pathogenic and can be transmitted sexually, by IDU, and from mother to child, persons infected with HTLV-1 should be given information regarding modes and efficiency of transmission, disease associations, and the probability of developing disease as described in detail elsewhere. ${ }^{2}$ While our study is the first to report HTLV infection in thalassemia patients from the United States, other reports from Greece and Iran have described HTLV rates of $1.1 \%$ and $6.5 \%$, respectively. ${ }^{7,8}$ In addition, HTLV-2 has been reported in $9.1 \%$ of Vietnamese thalassemia patients. ${ }^{9}$ Combined, these results further highlight the importance of this infection in this patient population, and suggest that HTLV testing should be considered in thalassemia patients, particularly if they were recipients of unscreened transfusions.

Our study has broader implications and supports further research to define HTLV prevalence in patients with other blood disorders that require chronic and potentially life-long blood transfusions, such as sickle cell anemia, bone marrow failure syndromes, myelodysplastic syndrome, and hereditary and acquired hemolytic anemias, especially if these patients were transfused in the United States before 1988. Since multiply transfused persons are potentially at higher risk of recognized or unrecognized blood-borne infections, it may

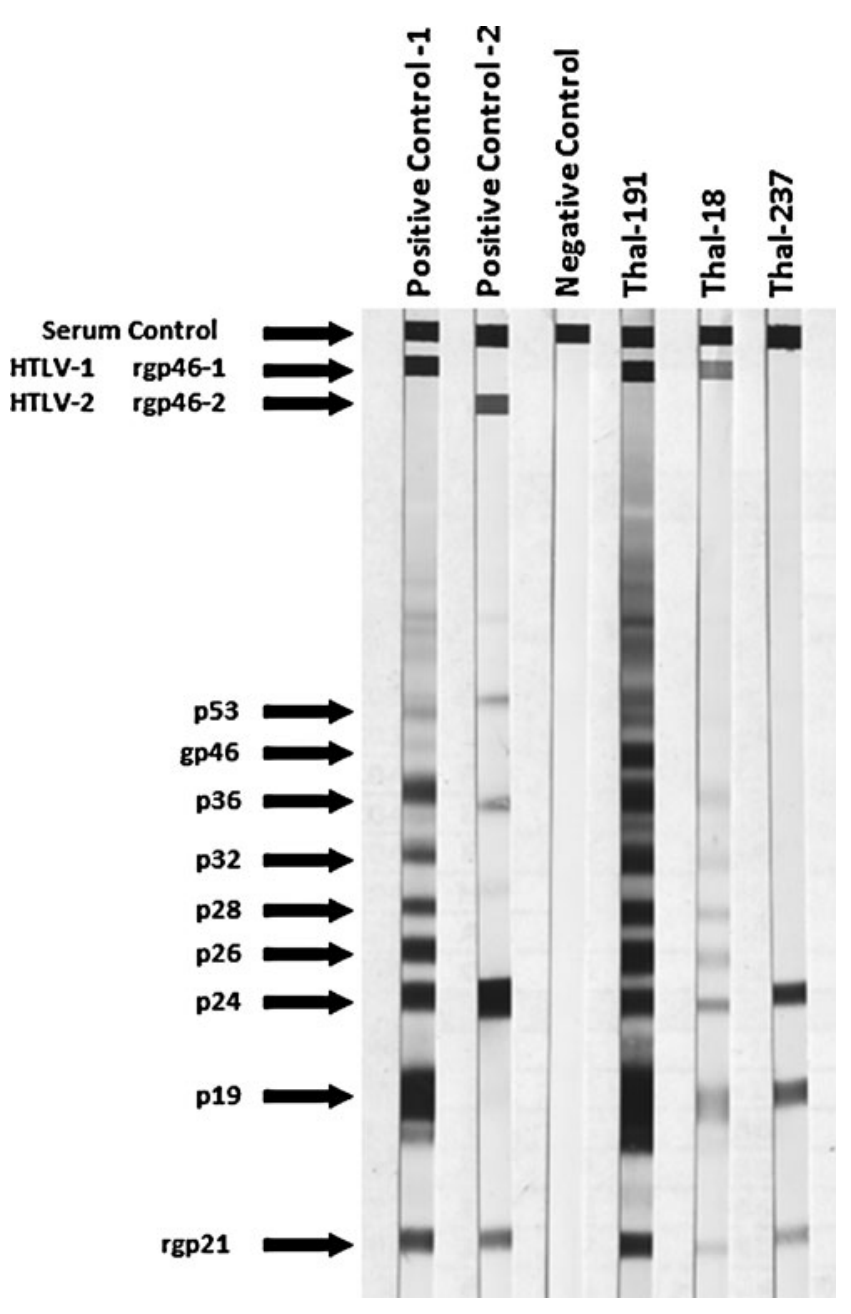

FIG. 1. Human T cell lymphotropic virus (HTLV) western blot profiles in U.S. thalassemia patients. Reactivity to HTLV-specific proteins is indicated on the left. Positive control 1 and 2 correspond to sera from HTLV-1-infected and HTLV-2-infected persons, respectively. Specimens that were reactive to the Gag (p24) and Env (GD21) proteins were considered seropositive. Seropositive specimens that were reactive to MTA-1 or K55 were considered HTLV-1-like or HTLV-2-like, respectively. Seropositive samples not reactive to either the MTA-1 or K55 peptides were considered HTLV positive, but untypeable. 
also be useful to consider the potential benefits of screening these patients for such agents by currently available metagenomics, microarray, and ultradeep sequencing technologies. ${ }^{10,11}$

\section{Acknowledgments}

Use of trade names is for identification only and does not imply endorsement by the U.S. Department of Health and Human Services, the Public Health Service, or the Centers for Disease Control and Prevention (CDC). The findings and conclusions in this report are those of the authors and do not necessarily represent the views of the CDC.

\section{Author Disclosure Statement}

No competing financial interests exist.

\section{References}

1. Beilke MA and Murphy EL: The human T-lymphotropic leukemia viruses 1 and 2. In: Viral and Immunological Malignancies (Volberding PA, Polensky J, eds.). BC Decker, Lewiston, NY, 2006.

2. Guidelines for counseling persons infected with human Tlymphotropic virus type I (HTLV-I) and type II (HTLV-II): Centers for Disease Control and Prevention and the U.S.P.H.S. Working Group. Ann Intern Med 1993;118(6): 448-454.

3. Sullivan MT, Williams AE, Fang CT, Grandinetti T, Poiesz BJ, and Ehrlich GD: Transmission of human T-lymphotropic virus types I and II by blood transfusion. A retrospective study of recipients of blood components (1983 through 1988). The American Red Cross HTLV-I/II Collaborative Study Group. Arch Intern Med 1991;151(10):2043-2048.

4. Martin F, Bangham CR, Ciminale V, et al.: Conference highlights of the 15th International Conference on Human
Retrovirology: HTLV and related retroviruses, 4-8 June 2011, Leuven, Gembloux, Belgium. Retrovirology 2011;8:86.

5. Zou S, Stramer SL, and Dodd RY: Donor testing and risk: Current prevalence, incidence, and residual risk of transfusion-transmissible agents in US allogeneic donations. Transfus Med Rev 2012;26(2):119-128.

6. Stramer SL, Notari EP, Zou S, et al.: Human T-lymphotropic virus antibody screening of blood donors: Rates of falsepositive results and evaluation of a potential donor reentry algorithm. Transfusion 2011;51(4):692-701.

7. Tseliou PM, Spanakis N, Spiliotakara A, Markogiannakis A, Legakis NJ, and Tsakris A: Prevalence of infection by HTLVI/II among pregnant women and high-risk groups in the Peloponnese peninsula, Greece. Int J Std Aids 2006; 17(8):543-546.

8. Karimi A and Nafisi M: Seroprevalence of human T-cell leukemia virus type 1 (HTLV-1) in high risk patients. J Res Health Sci 2006;6(1):44-47.

9. Lin MT, Nguyen BT, Binh TV, et al.: Human T-lymphotropic virus type II infection in Vietnamese thalassemic patients. Arch Virol 1997;142(7):1429-1440.

10. Delwart EL: Viral metagenomics. Rev Med Virol 2007; 17(2):115-131.

11. Barzon L, Lavezzo E, Militello V, Toppo S, and Palu G: Applications of next-generation sequencing technologies to diagnostic virology. Int J Mol Sci 2011;12(11):7861-7884.

Address correspondence to: William M. Switzer Laboratory Branch Division of HIV/AIDS Prevention Centers for Disease Control and Prevention 1600 Clifton Road, MS G-45 Atlanta, Georgia 30333

E-mail: bis3@cdc.gov 